\title{
Context and Text
}

\author{
Lihong Shen \\ English Department, Hebei United University, Tangshan, China; Communication University of China, Beijing, China \\ Email: fridaywhy@163.com
}

\begin{abstract}
Text and context are two essential elements in human communication. Text can not exist in a vacuum. Its production and comprehension are influenced and restricted by context. This paper aims to analyze the relations between context and text with the framework of both traditional context and cognitive context by Sperber \& Wilson.
\end{abstract}

Index Terms - context, cognitive context, text, utterance

\section{INTRODUCTION}

Language is the tool for communication. People use language to communicate with each other in society. By using language, they express their ideas, exchange their emotions and transform information about the world and the things in daily life, however, linguistic communication is not achieved by individual units of language, such as sounds, words or sentences. People, primarily and essentially, use combinations of these language units to express a whole idea in verbal communication. Text, as a linguistic term, is used to refer to these combinations. That is to say, a text can express the whole idea of the communicator. Brown and Yule (1983) in their Discourse Analysis define text as "the verbal record of a communicative act".

Text is produced and interpreted between two people in certain context, because human beings use language to communicate with others. According to the traditional view on context, context can be classified into co-text, situational context and cultural context, about which many linguists have done a lot of researches on the relations between text and context. In the linguistic communication, context determines text and text reflects context. (Ren Shaozeng, 1995)

The function of language is to organize people's common activities. One has to know how to say and what to say in order to communicate successfully. One has to understand why people say what they say and how they say it in a specific circumstance according to different aspects in the cognitive context. Different people and other factors will influence the producing and interpreting of the text.

\section{THE CONCEPTION OF CONTEXT}

In recent years, context has become the central topic of researches in the field of linguistics. Context is not something we find in Nature; context is a concept very used by philosophers and scientists, but with many different definitions, such that it would be better to speak of many different concepts, or at least a family-resemblance concept. Since Dummett we speak of "context principle" in Frege and Wittgenstein; we speak of "context of utterance" in pragmatics; we speak of "context sensitive" grammars in linguistics; and we speak also of "linguistic context" and "non-linguistic context" (Carlo Penco, 1999)

As such a highly ambiguous concept, context is hard to define. Though a lot of linguists have turned their eyes to the investigation of context in recent years, unfortunately, they have not been established a generally accepted and systematic theory about context standing by itself. As far as the categories of context are concerned, different scholars have different opinions. Basically, the categories of context can be divided into two kinds: one is the division within the same category; the other is the division of hierarchy. Different scholars utilize different names for the division of context. In a narrower sense, context consists of the lexical items that come immediately before and after any word in an act of communication. In a wider sense, everything may belong to a context, such as geographical and cultural background, the discourse interpretation and production in a certain communication, the discourse participants, their individual experiences, encyclopedic knowledge and their special roles in the communication, and the like.

In Changes and Development of Context Research, Qiu Xinyi puts forward seven key points for defining context:

i. Is context objective situation, psychological production or the background constructed by the communication subjects or not?

ii. Is context pre-existed before the communication or constructed dynamically during the process of a certain communication? Is it formed dynamically by the participants or by itself? The participants are constrained by context. Can they construct context for their communicative intention at the same time? Is the process accumulative if context is constructed continuously?

iii. What is context comparison with? Is it single or unique? Is it definite or not?

iv. Is context shared by the participants or included in their shared knowledge? Does context vary according to different participants? 
v. What level should we put context research on? Does an abstract and general context exist?

vi. What problems should we solve if we want to give a definition for context?

vii. What problems should we solve for constructing a descriptive context model?

All above seven aspects are theoretically valuable, scholars working in different disciplines tend to concentrate themselves on different aspects of context and hold diverse perspective or approaches.

\section{A BRIEF REVIEW OF CONTEXT RESEARCH}

\section{A. Traditional Research on Context}

The notion of the context is initiated by Malinowski, a British anthropology (born in Porland), in the complementary of The Sense of Sense by Ogden and Richards in 1923. He distinguished three types of context: the immediate context of utterance, the general context of situation, and the broader context of culture and put forward that context included not only the linguistic element but also cultural and situational factors.

Malinowski's notion of context of situation was accepted and elaborated by one of his colleagues Firth. He took over Malinowski's context of situation and extended it to linguistics. In 1950, he made a detail exposition about context in his book Personality and Language in Society. He further pointed out that Malinowski's conception of the context of situation was not quite adequate for the purpose of linguistic theory, because it was not general enough. Firth's own linguistic theory was built into by furthering the study of context. Four categories were proposed by him to cover the context of situation.

i. The participants in the situation: what Firth referred to person and personalities, corresponding more or less to what sociologists would regard as the status and roles of the participants;

ii. The action of the participants: what they are doing, including both their verbal and non-verbal action.

iii. Other relevant features of the situation: the relevant objects and non-verbal and non-personal events;

iv. The effects of the verbal action: what changes were brought about by what the participants in the situation had done.

$\mathrm{He}$ is the first to analyze language from the angle of context and sketch a framework for the study of context thereafter.

As one of Firth's students, M.A.K Halliday further develops the theory of situation into what is known as register theory, a more generalized interpretation intended as a basis for deriving the features of the text from the features of the situation. (Meiyun Yue, 1997).

The register theory is described in terms of a framework of three dimensions: field of discourse, tenor of discourse and mode of discourse. Field refers to the environment in which the speech event takes place which includes the topic, participants and the whole process of the interaction. It is the reflection of the social function of the text. Tenor is the relation between participants taking the social status and role into account. Tenor in effect directly determines the formality of the language used, and mode the medium of language activity. It is manifestation of the nature of the language code being used. In the other words, mode is the channel through which a language is used. Halliday gives us a specific and detailed analysis of context in discourse interpretation.

\section{B. Context and Pragmatics}

Leech (1983) defined pragmatics as "the study of how language is used in communication". In other words, pragmatics focuses on two maim topics: (1) what is communicated? (2) how is communicated ? As we have noted, communication does not take place in vacuum but in a context. Context is therefore an indispensable notion that makes pragmatics as it is. At present, the study of pragmatics mainly includes five aspects: deixis, conversational implicature, presupposition, speech acts and conversational structure. All these five aspects are actually involved in the problem of context. Since its great significant position as a corner stone to the discipline of pragmatics, pragmatic context is much broader in scope thus is much dwelt on by pragmatists.

Levinson is one of the pragmatists who are active on the scene of the newly arising discipline. His Pragmatics(1983) is a classic work which sketches the framework for the new discipline and is accepted by the later researcher ever since. In Pragmatics, the notion of context is not explicated separately in details but interrelated with other topics. In this book, Levinson (1983) claims that "context is understood to cover the identities of participants, the temporal and spatial parameters of the speech event, and the beliefs, knowledge and intentions of the participants in that speech event, and no doubt much besides." Levinson's context does not label all the actual situations of utterance in all their multiplicity of features, but only those features that are culturally and linguistically relevant to the production and interpretation of utterances. (van Dijk, 1976)

In Pragmatics, Levinson also discusses context in line with two socio-linguists J.Lyons and Ochs. Lyons considers that the following features should include the participants' knowledge of six aspects: i. knowledge of role and status (where role covers both the role in the speech event, as speaker or hearer, and the social role, and status cover notion of relative standing. ii. knowledge of spatial and temporal location; iii. knowledge of formality level; iv. knowledge of the medium(roughly the code or style appropriate to a channel, like the distinction between written and spoken varieties of a language); v. knowledge of appropriate subject matter; vi. knowledge of appropriate province (or domain determining the register of a language )(Levinson, 1983) Lyons' explanation of context can be regarded as an overlapping part as 
that of the pragmatics and thus takes it in. Ochs notes that the scope of context should embrace the social and psychological world in which the language user operates at any given time (Levinson, 1983). And that "context includes minimally language user's beliefs and assumptions about temporal, spatial and social settings; prior ongoing, and future actions (verbal and non-verbal), and the state of knowledge and attentiveness of those participating in the social interaction in hand" (cited in Levinson, 1983). Of course, Levinson also emphasizes that context does not exclude linguistic feature since such features often invoke contextual assumption.

One of the definitions about pragmatics put forward by Levinson is the study of the ability of language users to pair sentences with the contexts in which they would be appropriate (Levinson, 1983). From this we can see that context play a very important role in pragmatics. He also claims that:

For particular purpose, pragmatists are wont to restrict the nature of context in line with the problem in hand: thus in a work dealing mostly with presupposition and implicature, "contexts are set of propositions constrained only by consistency... The consistent sets of propositions that comprise contexts are to be interpreted as the unique speaker's own 'commitment slate' in the sense of Hamblin(1971)" (Gazdar,1979); while a work concerned with literary interpretation, "a context is construed as a 'complex event', viz. as an ordered pair of events of which the first causes the second. The first event is roughly the production of an utterance by the speaker, the second the interpretation of the utterance by the hearer" (van Dijk, 1976). But clearly a general theory of aspects of context relevant to production and interpretation must be broader than either of these.

An utterance, as he defines, is the issuance of a sentence, a sentence analogue, or sentence fragment, in an actual context (Levinson, 1983). So aspects of contexts play a very important role in the production and interpretation of the utterances.

Reviewing the ideas formulated by traditional researches, we seem to perceive a phenomenon: that is, when analyzing a text or a discourse, they normally believe context is set up in advance of comprehension. In much of the literature, it is explicitly or implicitly assumed that the context of a given utterance is not a matter of choice: the context is seen as determined, as given. Moreover, it is generally assumed that the context is determined in advance of the comprehension process.

\section{Cognitive Context}

With the analysis of traditional study of context, we seem to find the possibility of developing the research. Many scholars have stepped on this way. As we all know, communication by itself is dynamic so context can't be static for its important role in the communication. Compare with the traditional view on context, cognitive context is a dynamic one. In 1986, Sperber \& Wilson's works Relevance: Communication and Cognition came of the press and made a hit in the pragmatic circle. In this work, the co-authors note that a context is a psychological construct, a subset of the assumptions about the world. A context is not limited to information about the immediate physical environment or the immediately preceding utterances: expectations about the future, scientific hypotheses or religious beliefs, anecdotal memories, general cultural assumptions, beliefs about the mental state of the speakers, may all play a role in interpretation. (Sperber \& Wilson, 1995). It is these assumptions, rather than the actual state of the world that affect the interpretation of an utterance.

In verbal communication, significant to the interpretation of the utterance is not the immediate concrete environment but a series of assumptions that make up of the cognitive context. An individual forms an assumption in the expectation that he will be able to combine it with existing assumptions to derive a new assumption, which will yield what the co-author call a 'contextual effect'. According to their account of contextual effect, we have identified three ways in which a new item of information may have a contextual effect.

i. Strengthening existing assumptions;

ii. Contradicting with the existing assumptions;

iii. Combining with existing assumptions to yield contextual implications-conclusions derivable from input and context together, but from neither input nor context alone.

To Sperber \& Wilson, cognitive context is a fundamental concept. It is because of the diversity of the cognitive context that makes the interpretation of utterance difficult. Although some people live in the same physical world, deriving information from this common environment and constructing the best possible mental representation of it, no two individuals construct identical representation due to difference in many aspects especially in our cognitive abilities. Perceptual abilities vary in effectiveness from one person to another. Inferential abilities also vary, and not just in effectiveness. People speak different languages, they have mastered different concepts; as a result, they can construct different representations and make different inferences. (Sperber \& Wilson, 1995). Moreover, people have different memories that they bring to bear in their experience in different ways. Hence, even though they have the same physical environment, the cognitive environment would still differ. Cognitive environment is still not the genuine to context of communication but only a potential one needed to be filtered. Although people never share their total cognitive environments there is still intersection that is, there are a set of facts and assumptions manifest to them both. But this interesting part remains insignificant unless it is mutually manifest. That is to say, in a mutual cognitive environment, for every manifest assumption, the fact that it is manifest to the people who share this environment is itself manifest. (Sperber \& Wilson, 1995).

Sperber and Wilson's consideration of cognitive context differ dramatically from the traditional view on context 
which used to think that context keeps constant and exits in the minds of participants in advance. Traditional context is static, and cognitive context is dynamic, which is changing with the development of the communication. In this way, Sperber \& Wilson provides us a new angle of investigation of context and pragmatics.

\section{Structurization of Cognitive Context Factors}

Cognitive context is different from the traditional pragmatic context which consists of linguistic knowledge (the ability of mastering the language and knowledge about the co-text) and non-linguistic knowledge [background knowledge (encyclopedic knowledge, the social norms and conversational standards of certain culture); situational knowledge (time, place, topic, formal or informal occasion and relations between the participants); mutual knowledge of the participants]. However, what does cognitive context consist of? Xiong Xueliang (1999)cites van Dijk's argument 'context is in your mind' and he further puts forward that cognitive context, which involves various kinds of information: the perceptible information from outside; the information from long-term and short-term memory, refers to the conceptualized and sturcturized systematic pragmatic knowledge and is the result of the pragmatic factors internalized and recognized in people's mind, that is to say, the systemized pragmatic knowledge is stored in participants' mind and is activated to participate in the communication.

From cognitive point of view, the knowledge structure of human being is drawn from outside. The often-used language characters and context units can be structurized in the mind of different individuals. Liu Senlin (2000) has a study about the structurization of cognitive context.

He claims that the manipulation of cognitive context is based on the basic units called proposition, knowledge structure or mental schema. By this way, the original knowledge structures become the constituents of pragmatic logical inference, the original concrete context factors turn into various relations in the mind. Cognitive context comes into being by cognizing and structurizing these cognitive context factors.

According to structurization of cognitive context factors, these factors mainly include proposition, knowledge script, frame, mental schema and socio-psychological representation and so on. The theoretic basis of its structurization is from following theories, the theory of the modularity by Fodor, the theory of mental representation and proposition by Jackendoff, relevance theory by Sperber and Wilson and some theories about cognitive linguistics. Among them, relevance theory put forward by Sperber \& Wilson is the core theoretical basis of cognitive pragmatics and structurization of cognitive context factors. It embodies the combination of the modern cognitive science and pragmatics.

According to Relevance Theory, cognitive context is a psychological construct including not only the context of an utterance but also the contextual factors such as the immediate physical environment, the participant's background knowledge, the known facts, assumptions, beliefs, and cognitive abilities. It is a set of contextual assumptions that are stored in the brain of human being. But these assumptions are incomplete, a complete cognitive context can be formed only from inference which makes the participants achieve the pragmatic meaning in the variable communicative situations. Each assumption is not independent from each other since information is stored in the brain in the form of relevant group. (Ran Yongping, 2002) To some extent, we can say that all of these assumptions are structurized into such factors as schema, frame, knowledge script and socio-psychological representation etc.

These factors are called cognitive context models by van Dijk (1998) who claims that mental models also "play a role in the much neglected theory of discourse production, viz., as the mental point of departure of all text and talk, from which relevant information may be selected for the strategic construction of their global and local semantic structures."

Sperber and Wilson claim that a set of facts that an individual can understand construct cognitive context, as a result, these facts will influence the discourse production and interpretation. Cognitive context factors do more than act as interface between event models and semantic representations; they also seem to regulate the very structures of meaning.

\section{E. Relations between Traditional Context and Cognitive Context}

The essential distinction between the traditional context and cognitive context is that: traditional view considers that context is pre-existed and the interpretation of discourse depends on the encoding of language signal, while cognitive view argues that discourse interpretation depends on context which is formed by the assumptions under the general pragmatic principle. Through the process of psychological cognition, the assumptions construct new context to bring the explications and implications to light.

However, cognitive context and traditional context is not utterly opposite to each other. They are the same in essence. The traditional context may turn to cognitive context by the process of abstraction and internalization. After internalization, context is stored in the brain in the unit of knowledge script and psychological schema. (Zhou Guohui, 2005) We can say that cognitive context is a dynamic curve and the elements of the traditional context are the dots on it. In the process of communication, the communicative subjects will select some dots they need to construct a variable curve. The selected ones will be part of the cognitive context.

\section{TEXT/DiscoursE/UTTERANCE}

\section{A. Text and Discourse}

In daily life, people use language to communicate with each other. The individual units of language consist of sounds, 
words or sentences. However, people do not use these individual units for linguistic communication. People, primarily and essentially, communicate through combinations of these language units, which themselves constitute distinct units of expression. These combinations of language units are called texts in linguistics. But what is text?

About the definition of text, up till now there has not been a definite and perfect one in linguistic field. Different linguists have different opinions. According to Longman Dictionary of Language Teaching and Applied Linguistics, text is "a general term for example of language use, i.e. language which has been produced as the result of an act of communication" (Richard et al. 1989) Brown and Yule (1983) in their Discourse Analysis also define text as "the verbal record of a communicative act".

In Cohesion in English, Halliday \& Hasan define text as "any passage, spoken or written, of whatever length, that does form a unified whole" (Halliday \& Hasan, 1976). A text is a unit of language in use. It is not a grammatical unit, like a clause or a sentence; and it is not defined by its size. A text is sometimes envisaged to be some kind of super-sentence, a grammatical unit that is larger than a sentence but is related to a sentence in the same way that a sentence is related to a clause, a clause to a group and so on; by constituency, the composition of larger units out of smaller ones. But this is misleading. A text is not something that is like a sentence, only bigger; it is something that differs from a sentence in kind. It may be prose or verse, dialogue or monologue. It may be anything from a single proverb to a whole play, from a momentary cry for help to an all-day discussion on a committee. (Halliday \& Hasan, 1976). They also put forward the concept of texture, which can distinguish text and non-text. They claim that a semantically coherent text must have texture. If a passage containing more than one sentence is perceived as a text, there will be certain linguistic features present in that passage which can be identified as contributing to its total unity and giving it texture. Now compare the following examples:

(1) Mary is a good student. She is the first one in her class.

(2) Mary is a good student. It is getting warmer and warmer.

Obviously, (1) is a text because the second segment gives the relevant information and transforms a whole meaning that Mary is excellent. However, in example (2), both of the sentences are grammatically correct, but as a paragraph, it makes no sense for the segments are semantically incoherent. According to Halliday \& Hasan, it has no texture, so it can not be called a text.

In china, quite a number of scholars take different views concerning text. Huang Guowen(2001) elaborates his opinions about text in Theory and Practice of Discourse Analysis: A Study in Advertising Discourse:

Generally speaking, text can be defined from the angle of form, structure or function. In structure, text is a unit of language above the sentence. From linguistic point of view, the elements of language can be considered as the following hierarchical order: morpheme $\rightarrow$ word $\rightarrow$ group or phrase $\rightarrow$ clause $\rightarrow$ sentence $\rightarrow$ text. The latter is above the former. However, sometimes one word or even a morpheme can be called a text, for example, the signal "Exit" in the building or "Fire" when someone calls for help.

As function is concerned, text is language in use. According to different contexts, the meaning of the language varies. The same language unit has different meanings in different contexts. For example, the word "book" has different meanings in different occasions.

(3) He has written a book on discourse analysis. ("book" is a noun)

(4) I will book a room for you. (Here "book" is a verb, its meaning is reservation.)

From the systemic functional grammatical point of view, text is a language unit in use, a semantic unit. It is not a grammatical unit above a sentence. In fact, text and sentence do not belong to the same kind of linguistic unit. Strictly speaking, we can't say that text is above sentence, it is realized by sentences.

Hu Zhuanglin (1994) puts forward that text refers to the natural language in certain context, which is not bound by grammar but it can express complete semantics. In a word, according to the study about text in linguistics, the term text is used to refer to any passage, spoken of written, of whatever length, that does form a unified whole. Some regard it both as spoken and written language. Some (Coulthart, 1985) think that text only refers to written language, not including spoken. Leech (1983) employs the term discourse to refer to communicative language. But what is the difference between text and discourse?

From the above, we can find that discourse and text have been used in a variety of ways. In some cases the two terms have been treated as synonyms, while in others the distinction between discourse and text has been taken to apply to units of spoken versus written communication. Consequently, discourse analysis is, in some accounts, regarded as concerned with spoken texts. (Leech. 1983) Text, differently, has mainly been associated with written texts. We can say that the terms do not refer to different domains (speech and writing) but reflect a difference in focus. Discourse is the umbrella term for either spoken or written communication beyond the sentence. Text is the basic means of this communication, be it spoken or written, a monologue or an interaction. Discourse is thus a more embracing term that calls attention to the situated uses of text: it comprises both text and context. However, text is not just the products of discourse, as customarily assumed (Brown \& Yule, 1983) that is, the actual (written or spoken) language unit produced on the page. Text is the means of discourse, without which discourse would not be a linguistic activity.

According to Halliday \& Hasan(1976), a text is "a semantic unit: a unit not of form but of meaning". In their view, a text is "a semantic unit of language in use... Most texts extend well beyond the confines of a single sentence". Generally speaking, English and American linguistics tend to use the term discourse, and European scholars tend to use 
text.

In china, many scholars have different opinions about the term discourse and text. Hu Zhuanglin (1994) uses YUPIAN to refer to both text and discourse and claims that textlinguistics and discourse analysis is the same on the whole.

In the following discussion, we will not differentiate text from discourse strictly, we use these terms to refer to both spoken and written forms of language.

\section{B. Utterance}

Utterance is another linguistic term that we often touch upon in discourse analysis. Brown \& Yule (1983) make a distinction between utterance and sentence. They hold that utterance is segment of spoken language and sentence of written language. So comparing with sentence, utterance is much more flexible, it can be a word, a phrase, a sentence or a set of sentences and transforms a valuable communicative meaning in ordinary language-behavior. For example:

(5) A: Do you have classes on Saturday?

B: Yes.

(6) A: When did you stay with your aunt?

B: Over the weekend.

(7) A: Helen is a pretty girl?

B: I agree.

(8) A: Excuse me, can you tell me how to get to the Redland House Hotel?

B: Well, it's a bit complicated...um... I'd better show you on the map. It's on Kendal Street. Here it is. It will take about 20 minutes on foot, I think.

(5)B, (6) B,(7)B,(8)B can all be called utterances. Grice and Strawson use the term "utterance" to refer not just to linguistic utterance, or even to coded utterances, but to any modification of the physical environment designed by a communicator to be perceived by an audience and used as evidence of the communicative intentions.

From the cognitive point of view, linguistic communication is a process in which the communicative subjects employ the assumptions stored in the brain to transform and receive the information. A whole meaning can be considered as an information unit then every integrated information unit can be called as a text.

\section{CONCLUSION}

Communication, as we have noted, does not take place in a vacuum but in certain context, is a constant interactive process between the participants. Since the concept of context was initiated by Malinowski, different scholars have concentrated themselves on different aspects of contexts and hold diverse perspectives and approaches. Context is characterized by covering both the situational context and the linguistic context or be studied from a functional point of view by identifying three different aspects of context: field, tenor and mode. Context, as the most important notion in pragmatics, contributes a lot to discourse production and interpretation. The notion of context is not explicated separately in detail but interrelated with other topics to combine with the actual language use.

\section{REFERENCES}

[1] Brown, G. \& Yule, G. (1983). Discourse Analysis. New York: Cambridge University Press. 6-6.

[2] Carlo Penco. (1999). http://www.dif.unige.it/epi/hp/penco/pub/contr.htm (accessed 11/7/2012).

[3] Charles L. Hamblin. (1971) 'Mathematical Models of Dialogue', Theoria 37.2, 130-155.

[4] Coulthard, Malcolm. (1985). An Introduction to Discourse Analysis. London: Longman Group UK Limited.

[5] Fodor, J. A. (1983). The Modularity of Mind. Cambridge: MIT Press

[6] Gazdar' G. (1979). A solution to the projection problem. In C.K. Oh (ed.) Syntax \& Semantics 11: Presupposition. New York: Academic Press, 57-87.

[7] Guohui Zhou (2005) On Ambiguity of English and Chinese with the Framework of Cognitive Context, Foreign Language Education 26.2, 34-36.

[8] Grice, H.P. (1975). Logic and Conversation. In P. Cole \& J.L. Morgan (eds.) Syntax and Semantics: Speech Acts. New York: Academic Press. 41-58.

[9] Guowen Huang. (2001). Theory and Practice of Discourse Analysis. Shanghai: Shanghai Foreign Language Education Press

[10] Halliday, M.A.K. \& Hasan. R. (1976). Cohesion in English. London: Longman Group Ltd.

[11] Jackendoff. (1997). The Architecture of the Human Mind. Cambridge: MIT Press.

[12] Leech, G. (1983). Principles of Pragmatics. England: Longman Group UK Limited. 6,23,24.

[13] Levinson, S. (1983). Pragmatics. Cambridge: Cambridge University Press. 23-24.

[14] Meiyun Yue. (1997). Context and the Teaching of EFL Reading. Beijing: Qinghua University Press.

[15] Richards, J. C., Platt, J. and Platt, H. (1992). Longman Dictionary of Language Teaching and Applied Linguistics. Longman: Longman Group UK Limited. 83

[16] Shaozeng Ren. (1995).The Discourse Functions of Tense in English, Foreign Language, 3,22-29.

[17] Senlin Liu. (2000). On Structurization of Cognitive Environmental Factors, Journal of Sichuan International Studies University 16.4, 54-59

[18] Strawson, P. F. (1964). "Intention and Convention in Speech Acts." Philosophical Review 73, 439-60.

[19] Sperber, D. and D.Wilson.(1995). Relevance: Communication and Cognition. Oxford: Blackwell. 
[20] van Dijk, Teun A. (1976). "Pragmatics and Poetics." Pragmatics of Language and Literature, ed. Amsterdam: North-Holland.

[21] van Dijk, Teun A. (1998). Cognitive Context Models and Discourse. in Maxim Stamenov (ed), Language.189-226.

[22] Xueling Xiong. (1999). An Introduction to Cognitive Pragmatics. Shanghai: Shanghai Foreign Language Education Press.

[23] Xinyi Qiu. (1993). The Changes and Development of Context Studies. Rhetoric Learning 56.3, 4-6.

[24] Yongping Ran. (2002). A Relevance-theoretical Account of Politeness. Modern Foreign Languages 25.4, 387-395.

[25] Zhaoxiong He. (1999). An Introduction to Pragmatics. Shanghai: Shanghai Foreign Language Education Press.

[26] Zhuanglin Hu. (1994).Text Cohesion and Coherence. Shanghai: Shanghai Foreign Language Education Press.

Lihong Shen was born in Handan, China in 1978. She is a $\mathrm{PhD}$ candidate in linguistics in Communication University of China. She is currently an associate professor in the School of Foreign Languages, Hebei United University, Tangshan, China. His research interests include linguistics and literature. 